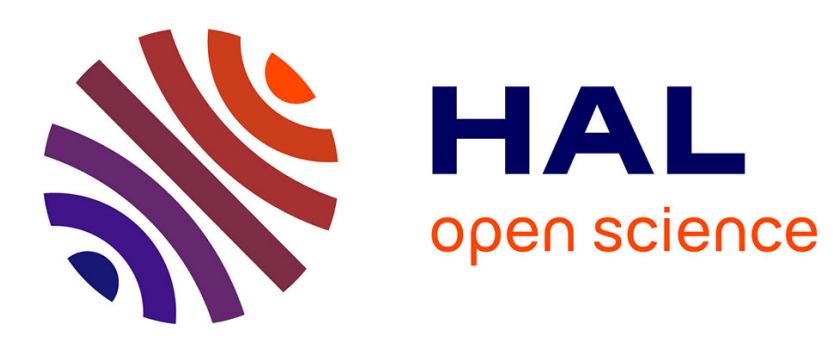

\title{
Caractérisation des écoulements turbulents instationnaires périodiques
}

Sedat F. Tardu

\section{To cite this version:}

Sedat F. Tardu. Caractérisation des écoulements turbulents instationnaires périodiques. Comptes rendus de l'Académie des sciences. Série IIb, Mécanique, 2003, 331 (11), pp.767-774. 10.1016/j.crme.2003.06.001 . hal-00261993

\section{HAL Id: hal-00261993 \\ https://hal.science/hal-00261993}

Submitted on 25 Feb 2020

HAL is a multi-disciplinary open access archive for the deposit and dissemination of scientific research documents, whether they are published or not. The documents may come from teaching and research institutions in France or abroad, or from public or private research centers.
L'archive ouverte pluridisciplinaire HAL, est destinée au dépôt et à la diffusion de documents scientifiques de niveau recherche, publiés ou non, émanant des établissements d'enseignement et de recherche français ou étrangers, des laboratoires publics ou privés. 


\title{
Caractérisation des écoulements turbulents instationnaires périodiques
}

\author{
Sedat Tardu \\ Laboratoire des écoulements géophysiques et industriels (LEGI), BP 53X, 38041 Grenoble cedex, France
}

\section{Résumé}

Le lien est établi entre les écoulements turbulents instationnaires à périodicité imposée et les processus cyclostationnaires (PCS) largement traités dans le domaine de la théorie de l'information. Les implications de ces propriétés dans la caractérisation des écoulements turbulents sont discutées, y compris pour des aspects de modélisation.

Mots-clés : Mécanique des fluides; Écoulements turbulents périodiquement forcés; Processus cyclostationnaires ; Caractérisation spectrale

\section{Abstract}

Characterization of unsteady time periodical turbulent flows. The link is established between turbulent unsteady flows, with imposed periodicity, and cyclostationary processes, often met in information theory. The implications of these properties for the characterization of turbulent flow is discussed, including the implications for aspects of modeling these flows.

Keywords: Fluid mechanics; Periodically forced turbulent flows; Cyclostationary processes; Spectral characterization

\section{Abridged English version}

Time periodical, forced unsteady flows (TPFUF) are encountered in several situations, such as turbulent channel flows with and without adverse pressure gradients with imposed velocity oscillations, turbulent boundary layers subject to local unsteady blowing/suction, pulsated jets, and to some extend turbulent wake flows. The classical characterization of these flows is based on the triple decomposition, called also phase averaging, that consists of considering separately the long time averages and cyclic modulations of the flow quantities. There is, however, a lack in the literature about the spectral characterization of these time non-equilibrium flows, together with alternate forms of representations.

Adresse e-mail : Sedat.Tardu@ @mg.inpg.fr (S. Tardu). 
The TPFUF are part of stochastic processes called cyclostationary in information theory. The nonstationary processes are characterized in a frequency plane, and their autocorrelation can be written as an extended double Fourier transform weighted by a frequency support function. The major property of the cyclostationnary processes with $T$ being the imposed oscillation period $(\operatorname{CSP}(T)$ ), is that their support consists of lines parallel to the diagonal and separated by $1 / T$. A number of important conclusions may then be deduced for the characterization of TPFUF. First of all, the long time averages resulting from phase averages correspond to the diagonal itself. Thus, any temporal invariant filtering that only covers the diagonal results in a stationary process. The cyclic modulations resulting from a low pass filtering with cut-off frequency smaller or equal than $1 / 2 T$, or a band pass filtering with bandwidth smaller than $1 / T$, are strictly zero. Thus, particular attention has to be paid for such processing when one deals with TPFUF. Indeed, time filtering is often used in turbulent flows to determine the characteristics of outer large scale (low-pass) and inner small scale (band-pass high-frequency cut-off) structures. For instance, the turbulent quantities related to structures resulting from a low pass filtering with cut-off frequency smaller than $1 / 2 T$ will not be modulated, not because they are 'frozen' and cannot follow the imposed unsteadiness, but simply because the filtering is not adequately defined. The outputs of disjoint filters in TPFUF are not null, contrarily to steady turbulent flows. Thus, a low-pass filtered signal that is subsequently band-pass-filtered in such a way that the bandwidths of the filters do not recover can present modulated statistics. Such interactions have to be taken into account in the physics of TPFUF.

The harmonic series representation of the $\operatorname{CSP}(T)$ is useful in many aspects. Each term of such series consists of a modulated stationary random process. These processes are conjointly stationary but not uncoupled. It is then possible to express the transport equations in terms of these time dependent stationary random functions and their interactions. Such representation may be useful in multiple scale, and rapid distortion models. It is finally asked whether the subclass of almost cyclostationary processes may be of use in the modelling of the presence of coherent structures, that produces the Reynolds shear stresses in a quasi-periodical way in time and space.

\section{Introduction}

On rencontre les écoulements turbulents instationnaires périodiques (ECTIP) dans de nombreuses situations : les écoulements pulsés soumis à des oscillations périodiques de vitesse avec ou sans gradient de pression (aérodynamique des pâles des hélicoptères, biomécanique, turbomachines, [1] et les références qui y sont citées), les couches limites turbulentes localement excitées par un soufflage/aspiration instationnaire périodique en temps, ou par un jet synthétique (contrôle actif de la turbulence pariétale et de la traînée, [2]), les jets pulsés (mélange et contrôle), les sillages turbulents dans certaines conditions etc. Le caractéristique commun de ces écoulements est la périodicité des moyennes statistiques. Une méthode classique de caractérisation consiste par conséquent à procéder par des moyennes de phase. Ainsi, une quantité $q$ est décomposée en une valeur moyenne $\bar{q}$, une composante oscillante $\tilde{q}$ de périodicité $T$ imposée, et une valeur fluctuante $q^{\prime}$. La moyenne de phase est définie par $\langle q\rangle=\bar{q}+\tilde{q}$. Parmi de nombreuses questions qu'on se pose à propos des écoulements non homogènes en temps, celle qui concerne la caractérisation spectrale vient en priorité. Les publications, existantes dans le domaine, ne traitent nullement cet aspect. Or, les ECTIP font partie des processus stochastiques cyclostationnaires. Ces derniers sont traités en grands détails dans la théorie de l'information depuis trois décennies (voir [3] pour une excellente revue). Le but de la présente note est de communiquer ces résultats d'une manière synthétique, et d'en tirer des conclusions pour les ECTIP avec la discussion qui s'impose.

\section{Particularités essentielles des processus cyclostationnaires et leurs implications}

Un processus $x(t)$ est harmonisable, s'il peut s'écrire sous la forme :

$$
x(t)=\int_{R} \exp (\mathrm{i} \lambda t) Y(\mathrm{~d} \lambda)
$$


où $Y$ est une mesure aléatoire au sens de Loève [4,5]. La fonction d'autocorrélation de $x(t)$ s'écrit alors :

$$
r_{x x}(s, t)=\int_{-\infty}^{\infty} \int_{-\infty}^{\infty} \exp [\mathrm{i} 2 \pi(s f-t v)] r_{Y}(\mathrm{~d} f, \mathrm{~d} \nu)
$$

où $r_{Y}(\mathrm{~d} f, \mathrm{~d} v)$ peut être interprété comme la zone du domaine fréquentiel $f \times v$, dans laquelle l'énergie du processus est concentrée (voir [4], p. 57, pour une définition plus rigoureuse). On appellera $r_{Y}(\mathrm{~d} f, \mathrm{~d} \nu)$ le support de $x(t)$.

Le processus $x(t)$ est cyclostationnaire avec périodicité $T$ - PCS $(T)$ par la suite - si sa corrélation est périodique :

$$
r_{x x}(s, t)=E\left[x(s) x^{*}(t)\right]=r_{x x}(s+m T, t+n T)
$$

où $m$ et $n$ sont deux entiers, $E$ indique l'espérance mathématique et $(*)$ est le conjugué complexe. La périodicité implique :

$$
r_{x x}(s, t)=\frac{1}{2 N+1} \sum_{k=-N}^{k=N} r_{x x}(s+k T, t+k T)
$$

(2) combiné avec (4) permet d'écrire :

$$
r_{x x}(s, t)=\int_{-\infty}^{\infty} \int_{-\infty}^{\infty} \exp [\mathrm{i} 2 \pi(s f-t v)] D_{N}(f, v) r_{Y}(\mathrm{~d} f, \mathrm{~d} v)
$$

avec :

$$
D_{N}(f, v)=\frac{1}{2 N+1}\left\{1+2 \sum_{k=1}^{k=N} \cos [2 \pi k T(f-v)]\right\}
$$

L'identité des Éqs. (2) et (5) entraîne $D_{N}(f, v)=1$, ce qui n'est possible que si et seulement si $f=v-j / T$, $j$ étant n'importe quel nombre entier. Ce résultat constitue une propriété essentielle des $\operatorname{PCS}(T):$ le support $r_{Y}(\mathrm{~d} f, \mathrm{~d} v)$ d'un $\operatorname{PCS}(T)$ est restreint à des lignes parallèles à la diagonale $f=v$ et distantes de $1 / T$ comme le montre la Fig. 1. Cette propriété est importante pour l'interprétation physique des caractéristiques des écoulements turbulents à périodicité imposée :

1. Le support de la partie stationnaire, ou, en termes de moyenne de phase discutée dans l'introduction, de la moyenne de $r_{x}(s, t)$ est la diagonale $f=v$. On a en fait :

$$
\overline{r_{x x}}(s, t)=\overline{r_{x x}}(s-t)=\int_{-\infty}^{\infty} \int_{-\infty}^{\infty} \exp [\mathrm{i} 2 \pi f(s-t)] r_{Y}(\mathrm{~d} f, \mathrm{~d} f)
$$

Le support de la modulation de $\tilde{r}_{x}(s, t)$, par conséquent, est l'ensemble des droites $f=v-j / T$ avec $j \neq 0$ c.à.d. excepté la diagonale $f=v$.

2. La Fig. 1 montre clairement que tout filtrage passe bas avec une fréquence de coupure inférieure à $1 /(2 T)$ résulte en un signal stationnaire, car le domaine du filtrage ne recouvre que la diagonale $f=v$. La sortie d'un filtre passe bande dont l'entrée est un $\operatorname{PCS}(T)$ et de bande passante inférieure à $1 / T$ donne également lieu à un signal stationnaire pour la même raison (Fig. 1). On a souvent recours dans les écoulements turbulents, au filtrage temporel passe-bas et passe-bande pour déterminer les caractéristiques des structures à grandes et petites échelles [6]. Ces structures ont respectivement des comportements passifs et actifs dans le cadre des écoulements turbulents pariétaux. Leur réponse aux excitations périodiques forcées de vitesse sont déterminées par la moyenne de phase des intensités turbulentes des signaux temporellement filtrés [7]. Le choix des 


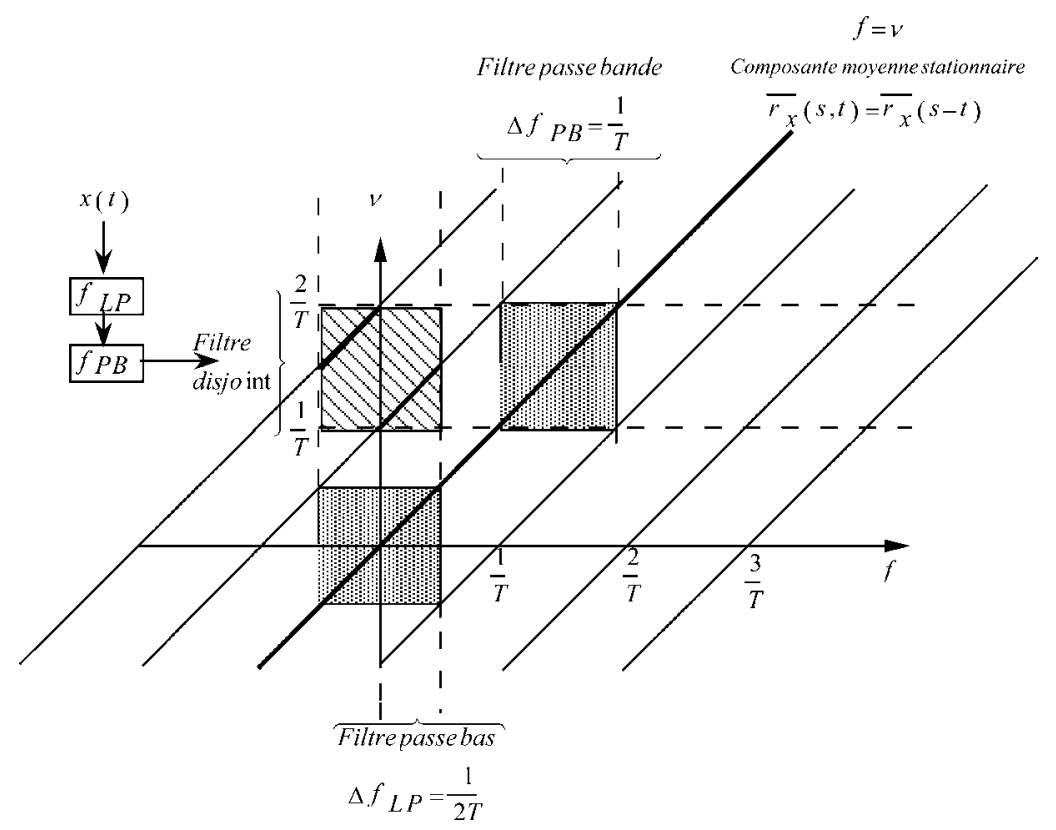

Fig. 1. Le support des $\operatorname{PCS}(T)$ consiste en des lignes parallèles à la diagonale et séparées de $1 / T$.

Fig. 1. The spectral support of cyclostationary processes is constrained to the lines parallel to the diagonal and separated by $1 / T$.

filtres avec des bandes passantes qui ne font passer que la partie stationnaire peut alors donner lieu à des interprétations erronées. Ainsi, un filtrage passe bas avec $\Delta f_{L P} \leqslant 1 /(2 T)$ va naturellement donner lieu aux modulations nulles des intensités turbulentes, non parce que ces structures sont physiquement gelées durant la période d'oscillation imposée, mais tout simplement parce que le choix de la bande passante a été mal fait au départ.

Ce fait est illustré par la Fig. 2 qui montre les amplitudes relatives des fluctuations de vitesse longitudinale $\left\langle u^{\prime} u^{\prime}\right\rangle$ à une distance $y^{+}=10$ de la paroi, dans un écoulement turbulent en canal assujetti à des oscillations périodiques forcées de vitesse, en fonction de la fréquence imposée $f^{+}[1]$ (+ indique les variables adimensionalisés par rapport à la vitesse de frottement et la viscosité cinématique). L'amplitude des oscillations est $20 \%$ de la vitesse au centre du canal. L'amplitude relative $a_{\tilde{q}}$ liée à la quantité $q$ est le rapport de l'amplitude de la fondamentale $A_{\tilde{q}}$, à la valeur moyenne temporelle $\bar{q}$. L'amplitude relative $a_{u^{\prime} \tilde{u}^{\prime}}$ est comparée avec l'amplitude des signaux filtrés passe bande. Filtre 1 a une bande-passante de $\delta f^{+}=0,0055-0,022$ avec $\Delta f_{P B}^{+}=0,0165$ et détecte les structures actives près de la paroi [6]. On voit que la bande passante du filtre 1 satisfait la condition $\Delta f_{P B}^{+}>1 / T^{+}$dans toute la gamme de fréquence imposée et que le filtrage recouvre la partie oscillante du support spectral. Le filtre A (carrés dans la Fig. 2), en revanche, a une bande passante $\Delta f_{P B}^{+}=0,003$ plus étroite $\left(\delta f^{+}=0,01-0,013\right)$ et il y a extinction de la modulation pour $f^{+}>0,003$. La méconnaissance des caractéristiques cyclostationnaires conduirait à conclure que les structures associées au filtre A ont un temps de relaxation plus grand, mais tel n'est bien évidemment pas le cas.

3. La sortie d'un filtre disjoint dont l'entrée est un $\operatorname{PCS}(T)$ n'est pas nulle comme c'est le cas d'un signal stationnaire aléatoire. On entend par filtrage disjoint, l'opération qui consiste à filtrer un signal consécutivement par deux filtres dont les bandes passantes ne cö̈ncident pas. Un exemple est donné sur la Fig. 1. Le signal est d'abord filtré par le filtre passe-bas avec $\Delta f_{L P}=1 /(2 T)$ et passe ensuite à travers le filtre passe bande défini sur $\rfloor 1 / T, 1 /(2 T)\lfloor$. On voit que la zone hachurée de filtrage disjoint contient des segments des droites (mis en gras sur la figure) $v=f+1 / T$ et $v=f+2 / T$. Les moyennes de phases des intensités turbulentes des signaux disjointement filtrés peuvent donc être modulées bien que leur moyennes soient nulles. 


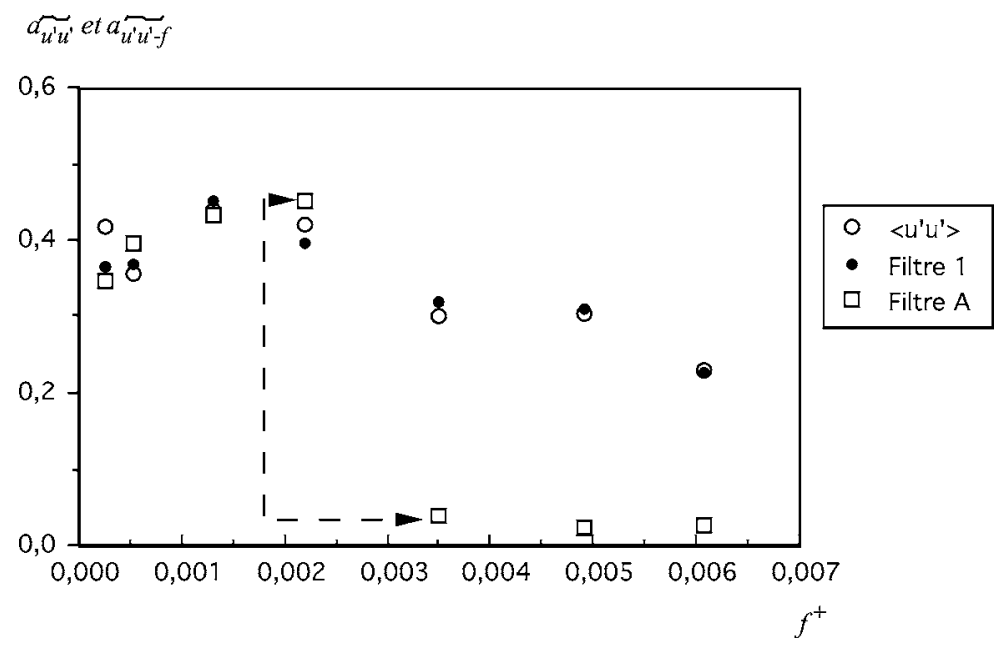

Fig. 2. Les amplitudes relatives à $y^{+}=10$, de la modulation de l'intensité turbulente longitudinale et de la vitesse temporellement filtrée dans un écoulement en canal pleinement turbulent assujetti à des oscillations de vitesse en fonction de la fréquence imposée $f^{+}$. Le filtre 1 a une bande passante qui recouvre le support oscillant. La bande passante du filtre A, en revanche, est inférieure à 0,003. L'extinction de l'amplitude correspondante, indiquée par les flèches, est totalement arbitraire et n'a pas de sens physique.

Fig. 2. The relative amplitudes of the longitudinal turbulent intensity modulations and pass band filtered signals at $y^{+}=10$ in a fully developed turbulent channel flow subject to imposed velocity oscillations versus the imposed frequency $f^{+}$. The pass band of the filter 1 recovers entirely the oscillating support. The pass band of filter A, in return, is smaller than 0.003 . The suppression of the oscillations shown by the arrows is entirely arbitrary and does not have a physical meaning.

\section{Représentation en série harmonique}

Tout processus harmonisable peut être représenté par une série $x(t)=\sum_{-\infty}^{\infty} x_{k}(t)$ où $x_{k}(t)$, également harmonisable, est la sortie d'un filtre passe bande défini sur l'intervalle $E_{k}=\left[\lambda_{k}, \lambda_{k+1}\right]$ dont l'union $\bigcup E_{k}=R$ est l'ensemble des réels [4]. Le choix $E_{k}=[2 k / T, 2(k+1) / T]$ s'impose naturellement pour un PCS $(T)$ ce qui permet d'écrire :

$$
\begin{aligned}
x(t) & =\sum_{-\infty}^{\infty} x_{k}(t)=\sum_{-\infty}^{\infty} \int_{2 k / T}^{2(k+1) / T} \exp (\mathrm{i} \lambda t) Y(\mathrm{~d} \lambda) \\
& =\sum_{-\infty}^{\infty}\left[\int_{0}^{2 / T} \exp (\mathrm{i} \lambda t) Y\left(\mathrm{~d} \lambda+\frac{2 k}{T}\right)\right] \exp \left(\frac{\mathrm{i} 2 \pi k t}{T}\right)=\sum_{-\infty}^{\infty} a_{k}(t) \exp \left(\frac{\mathrm{i} 2 \pi k t}{T}\right)
\end{aligned}
$$

Comme $a_{k}(t) \exp (\mathrm{i} 2 \pi k t / T)$ est la sortie du filtre passe bande :

$$
W_{k}(f)=1 \quad \text { si }\left|f-\frac{k}{T}\right| \leqslant \frac{1}{2 T}
$$

dont l'entrée est $x(t)$, il est facile de montrer que les coefficients temporels $a_{k}(t)$ sont les sorties du filtre $W_{0}(f)$, lorsque l'entrée est $x(t) \exp (-\mathrm{i} 2 \pi t / T)$. Ceci s'établit facilement en utilisant les relations d'entrée et de sortie des systèmes linéaires, comme le montre schématiquement la Fig. 3. Etant donnée que la fonction de transfert de $W_{0}(f)$ n'est autre que $w(t)=\sin (\pi t / T) /(\pi t)$, on obtient :

$$
a_{k}(t)=\int_{-\infty}^{\infty} w(t-\tau) x(\tau) \exp \left(-\frac{\mathrm{i} 2 \pi k \tau}{T}\right) \mathrm{d} \tau
$$




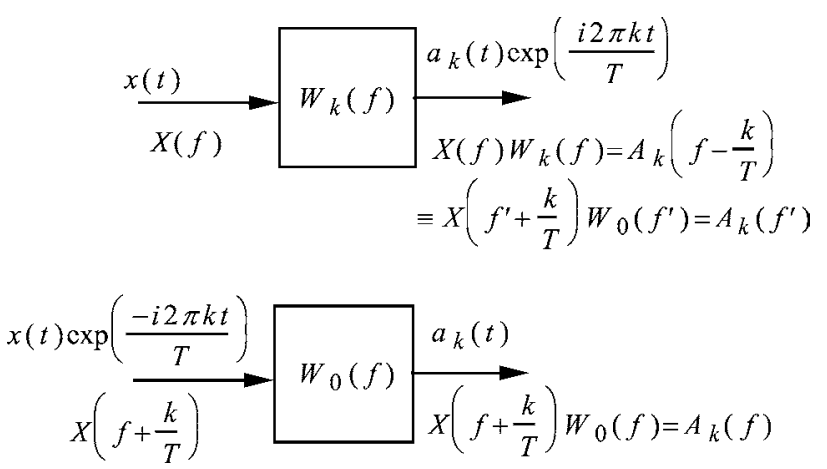

Fig. 3. Décomposition en série harmonique du signal cyclostationnaire $x(t)$ à travers les filtres $W_{k}(f)$ définis dans le texte. Les symboles en majuscule correspondent aux transformées de Fourier. Les deux systèmes sont équivalents par translation.

Fig. 3. The decomposition of the cyclostationary process $x(t)$ into stationary components via the filtres $W_{k}(f)$ defined in the text. The capital symbols correspond to the Fourier transforms of the variables. Both systems shown in the figure are equivalent, by translation.

Il y a une propriété intéressante et importante des fonctions aléatoires $a_{k}(t)$ pour les processus cyclostationnaires : les $a_{k}(t)$ sont conjointement stationnaires si et seulement si $x(t)$ est $\operatorname{CS}(T)[3,4]$. Ceci permet de caractériser différemment les écoulements turbulents à périodicité imposée, comme on en discutera par la suite. La représentation en série permet d'écrire :

$$
r_{x x}(s, t)=\sum_{k, l=-\infty}^{\infty} r_{k l}(s, t) \exp \left[\frac{\mathrm{i} 2 \pi(k s-l t)}{T}\right]
$$

pour la fonction d'autocorrélation $r_{x x}(s, t)$ où $r_{k l}(s, t)=E\left(a_{k}(s) a_{l}^{*}(t)\right)$ est la fonction d'autocorrélation concernant les fonctions $a_{k}(t)$. En utilisant (9), on a :

$$
r_{k l}(s, t)=\int_{-\infty}^{\infty} \int_{-\infty}^{\infty} w(s-\tau) w(t-\gamma) r_{x x}(\tau, \gamma) \exp \left[\frac{-\mathrm{i} 2 \pi(k \tau-l \gamma)}{T}\right] \mathrm{d} \tau \mathrm{d} \gamma
$$

En exprimant les fonctions de transfert par leur transformée de Fourier inverse et en remplaçant dans (11), on obtient :

$$
r_{k l}(s, t)=\int_{-1 / 2 T}^{1 / 2 T} \int_{-1 / 2 T}^{1 / 2 T} R_{x x}\left(f+\frac{k}{T}, v+\frac{l}{T}\right) \exp [\mathrm{i} 2 \pi(f s-v t)] \mathrm{d} f \mathrm{~d} v
$$

ce qui se réduit pour les $\operatorname{PCS}(T)$ à :

$$
r_{k l}(s, t)=\int_{-1 / 2 T}^{1 / 2 T} \int_{-1 / 2 T}^{1 / 2 T} R_{x x}\left(f+\frac{k}{T}, v+\frac{l}{T}\right) \exp [\mathrm{i} 2 \pi f(s-t)] \mathrm{d} f \mathrm{~d} v
$$

qui montre que $r_{k l}(s, t)=r_{k l}(s-t)$ ne dépend que du décalage $s-t$, et c'est la raison pour laquelle les processus $a_{k}(t)$, sont conjointement stationnaires.

La moyenne de phase $\langle x x\rangle$ peut s'exprimer en fonction des corrélations croisées $r_{k l}(s, t)=E\left(a_{k}(s) a_{l}^{*}(t)\right)$. La moyenne d'ensemble n'est autre que $\langle x x\rangle=r_{x x}(t, t)$ et d'après l'Éq. (10) et la stationnarité des $a_{k}(t)$ :

$$
\langle x x\rangle=\sum_{k, l=-\infty}^{\infty} r_{k l}(t, t) \exp \left[\frac{\mathrm{i} 2 \pi t(k-l)}{T}\right]=\sum_{k, l=-\infty}^{\infty} r_{k l}(0) \exp \left[\frac{\mathrm{i} 2 \pi t(k-l)}{T}\right]
$$


La valeur moyenne temporelle $\overline{x x}$ s'obtient pour $k=l$ :

$$
\overline{x x}=\sum_{k=-\infty}^{\infty} r_{k k}(0)=\sum_{k=-\infty}^{\infty} E\left(a_{k}(t) a_{k}^{*}(t)\right)
$$

autrement dit, par la somme de l'énergie des signaux stationnaires $a_{k}(t)$. Il suffit, afin de montrer cette dernière égalité, de modifier l'Éq. (13) en :

$$
r_{k k}(0)=\int_{-1 / 2 T+k / T-1 / 2 T+k / T}^{1 / 2 T+k / T} \int_{x x}^{1 / 2 T+k / T} R_{x}(f) \mathrm{d} f \mathrm{~d} v
$$

et se rappeler que le support de $x(t)$ est tel que $v=f-j / T$. L'intégrant de (16) ne recouvre que la partie stationnaire et, par conséquent, la somme dans (15) est bien la valeur moyenne temporelle $\overline{x x}$. La partie oscillante $\widetilde{x x}$ est liée aux corrélations strictement croisées $r_{k l}(0)$ avec $k \neq l$ :

$$
\widetilde{x x}=\sum_{\substack{k, l=-\infty \\ k \neq l}}^{\infty} r_{k l}(0) \exp \left[\frac{\mathrm{i} 2 \pi t(k-l)}{T}\right]
$$

On peut alors suggérer une procédure intéressante pour la caractérisation des écoulements turbulents instationnaires à périodicité imposée. Il s'agit de déterminer les signaux stationnaires $a_{k}(t)$ par filtrage passe bas du signal $x(t) \exp (-\mathrm{i} 2 \pi k t / T)$ à travers le filtre $W_{0}(f)$, et de déterminer les spectres $A_{k}(f)$ par des méthodes conventionnelles. La détermination des $A_{k}(f)$ caractérise complètement le $\operatorname{PCS}(T)$. Le nombre $N_{a}$ des fonctions aléatoires $a_{k}(t)$ est limitée par la fréquence de Kolmogoroff $f_{K}$ du processus global et $N_{a}=T f_{K}$. Il y a évidemment certaines questions qui restent ouvertes à l'heure actuelle. Trouvera-t-on le cascade classique dans les spectres $A_{k}(f)$ ? Si oui, est-ce que tous les spectres présenteront une zone inertielle (par exemple), ou le comportement en $-5 / 3$ (pour ne citer que celui-ci) apparaitra-t-il plutôt lorsque $k$ est suffisamment grand, puisque les coefficients $a_{k}(t) \exp (-\mathrm{i} 2 \pi k t / T)$ se rapprocheraient alors davantage des petites échelles ? Les réponses à ces questions dépendent certainement de l'écoulement et de l'instationnarité imposée.

\section{4. Équations de transport}

La représentation en série harmonique permet d'obtenir des interrelations entre les corrélations des composantes stationnaires $a_{k}(t)$ reliées aux quantités gouvernant les équations de transport. A titre d'exemple, prenons l'équation de transport de la moyenne de phase de la contrainte de Reynolds $\left\langle u^{\prime} v^{\prime}\right\rangle$ dans un écoulement turbulent cisaillé, périodiquement forcé, homogène dans les directions longitudinales et transversales $x$ et $z$ et non homogène suivant $y$. En passant par les représentations en série harmoniques des composantes de vitesse fluctuante :

$$
u^{\prime}(t, \vec{x})=\sum_{k=-\infty}^{k=\infty} u_{k}(t, \vec{x}) \exp \left(\frac{\mathrm{i} 2 \pi k t}{T}\right), \quad v^{\prime}(t, \vec{x})=\sum_{l=-\infty}^{l=\infty} v_{l}(t, \vec{x}) \exp \left(\frac{\mathrm{i} 2 \pi l t}{T}\right)
$$

où, $\vec{x}$ indique que les quantités dépendent non seulement du temps, mais également de l'espace, la moyenne de phase de $\left\langle u^{\prime} v^{\prime}\right\rangle$ s'écrit comme :

$$
\left\langle u^{\prime} v^{\prime}\right\rangle=r_{u^{\prime} v^{\prime}}(t, t)=\sum_{k, l=-\infty}^{\infty} r_{u_{k} v_{l}}(0, y) \exp \left[\frac{\mathrm{i} 2 \pi t(k-l)}{T}\right]
$$

où on précise que $r_{u_{k} v_{l}}(0, y)=E\left(u_{k}(t, y) v_{k}^{*}(t, y)\right)$. En introduisant également la pression fluctuante $p^{\prime}(t, \vec{x})=$ $\sum_{-\infty}^{\infty} p_{m}(t, \vec{x}) \exp (\mathrm{i} 2 \pi m t / T)$ on peut aisément arriver à l'égalité suivante, déduite de l'équation de transport de $\left\langle u^{\prime} v^{\prime}\right\rangle$ normalisée par une échelle de vitesse convenable et de la viscosité : 


$$
\begin{aligned}
\frac{\mathrm{i} 2 \pi(k-l)}{T} r_{u_{k} v_{l}}= & -r_{v_{k} v_{l}} \frac{\partial\langle U\rangle}{\partial y}+\frac{\partial^{2} r_{u_{k} v_{l}}}{\partial y^{2}}+\left[r_{p_{k} \partial v_{l} / \partial x}+r_{p_{k} \partial u_{l} / \partial y}-\frac{\partial r_{p_{k} u_{l}}}{\partial y}\right] \\
& -\frac{\partial r_{p_{k} v_{l}^{2}}}{\partial y}-2\left[r_{\partial u_{k} / \partial x \partial v_{l} / \partial x}+r_{\partial u_{k} / \partial y \partial v_{l} / \partial y}+r_{\partial u_{k} / \partial z \partial v_{l} / \partial z}\right]
\end{aligned}
$$

Le terme à gauche de l'équation précédente provient de $\partial\left\langle u^{\prime} v^{\prime}\right\rangle / \partial t$. Les termes à droite sont regroupés dans l'ordre : le premier résulte du terme de production $-\left\langle v^{\prime} v^{\prime}\right\rangle \partial\langle U\rangle / \partial y$, le deuxième de la diffusion visqueuse $D=\partial^{2}\left\langle u^{\prime} v^{\prime}\right\rangle / \partial y^{2}$, le troisième groupe entre crochets est l'expression de corrélation pression-vitesse $\Pi=$ $\left\langle p^{\prime} \partial v^{\prime} / \partial x\right\rangle+\left\langle p^{\prime} \partial u^{\prime} / \partial y\right\rangle-\partial\left\langle u^{\prime} p^{\prime}\right\rangle / \partial y$, le quatrième provient de la diffusion turbulente $T=-\partial\left\langle u^{\prime} v^{\prime 2}\right\rangle / \partial y$ et le dernier de la dissipation $\varepsilon=-2\left\langle\partial u^{\prime} / \partial x_{j} \partial v^{\prime} / \partial x_{j}\right\rangle$.

On remarque que la forme de l'équation de transport de $\partial\left\langle u^{\prime} v^{\prime}\right\rangle / \partial t$ reste identique dans sa représentation en série, pour les écoulements turbulents à périodicité imposée. La dérivée par rapport au temps $\partial\left\langle u^{\prime} v^{\prime}\right\rangle / \partial t$ s'annule pour les composantes $k=l$ qui correspondent à la partie stationnaire, ou en d'autres termes, à la moyenne temporelle $\overline{u^{\prime} v^{\prime}}$ pour des raisons invoquées à la fin de la section précédente. Ces résultats peuvent être facilement généralisés pour les autres composantes du tenseur des contraintes de Reynolds telles que $\partial\left\langle u^{\prime} u^{\prime}\right\rangle / \partial t$ et $\partial\left\langle v^{\prime} v^{\prime}\right\rangle / \partial t$. La modélisation des équations de transport exprimées sous cette forme reste cependant un challenge à l'heure actuelle, bien qu'elle paraisse, au premier abord, mieux adaptée aux modèles à échelles multiples, ou à distorsion rapide [8].

\section{Conclusion}

Les écoulements turbulents instationnaires périodiques en temps ont des caractéristiques qui peuvent être fondamentalement différents comparés aux écoulements turbulents temporellement homogènes. Il faut en particulier être vigilant vis-à-vis des quantités découlant des filtrages temporels. Nous allons faire une dernière remarque pour conclure. L'importance des structures cohérentes actives qui contribuent majoritairement aux contraintes de Reynolds est un fait parfaitement établi à l'heure actuelle. Ces structures se régénèrent quasipériodiquement dans l'espace et le temps. L'investigation des processus quasi-cyclostationnaires analysés par [9] peut être d'une utilité certaine pour des aspects de modélisation des écoulements turbulents à cohérence marquée.

\section{Références}

[1] S. Tardu, G. Binder, R.-F. Blackwelder, Turbulent channel flow subjected to large imposed velocity oscillations, J. Fluid Mech. 267 (1994) $109-151$

[2] S. Tardu, Active control of near wall turbulence by local unsteady blowing, J. Fluid Mech. 43 (2001) 217-253.

[3] W.-A. Gardner, L.-E. Franks, Characterization of cyclostationary random signal processes, IEEE Trans. Inform. Theory IT-21 (1975) 4-14.

[4] H.L. Hurd, Representation of strongly harmonizable periodically correlated processes and their covariances, J. Multivariate Anal. 29 (1989) 53-67.

[5] H. Ogura, Spectral representation of a periodic nonstationary random process, IEEE Trans. Inform. Theory IT-17 (1971) $143-149$.

[6] A.-M. Naguib, C.-E. Wark, An investigation of wall-layer dynamics using a combined temporal filtering and correlation technique, J. Fluid Mech. 243 (1992) 541.

[7] P. Vezin, S. Tardu, G. Binder, Corrélations spatio-temporelles et échelles de longueur dans les écoulements pulsés en canal, Rapport de Synthèse Finale $\mathrm{n}^{\mathrm{o}}: 91.086,1994$.

[8] S. Tardu, P. Da Costa, Modeling of unsteady wall flows with and without adverse pressure gradient by a $k-\omega$, Rapid distorsion closure, in : Second International Turbulent Shear Flow Phenomena Symp., Stockholm, Juin 27-29, 2001.

[9] W.-A. Gardner, Stationarizable random processes, IEEE Trans. Inform. Theory IT-24 (1978) 8-22. 